\title{
Avis de recherche
}

Claude Daviau

doi:10.10.18162/fp.2018.a156

Commission scolaire de Montréal

\section{HRONIQUE • Milieu scolaire}

Dans la perspective de l'article Créer un espace de partage des expériences de collaboration entre les milieux universitaire et scolaire en vue de l'amélioration des pratiques pédagogiques et de gestion en éducation (Fejzo, Dufour et Daviau, 2018), le présent propos se situe comme étant une réflexion personnelle, sans prétention, relative au domaine de la recherche en général, et plus spécifiquement des besoins du chercheur au regard de ceux du praticien, des retombées des données probantes parfois difficiles à implanter, de l'accès aux résultats dans tous les milieux et de la collaboration entre les institutions universitaires, les établissements et les commissions scolaires ainsi que leurs différents acteurs. Ce point de vue et les constats qui le sous-tendent font référence à un parcours diversifié d'expériences et d'expertise distribuées dans le temps entre l'actualisation des rôles d'enseignant, de conseiller pédagogique, de direction d'établissement, de coordonnateur des ressources éducatives et informatiques, de direction d'un réseau d'établissements primaires et secondaires, de négociateur de convention collective et finalement de direction générale adjointe d'une commission scolaire. Bref, une opportunité de partager un vécu expérientiel en éducation, échelonné sur 32 années et des poussières, empreint de recherches sans pour autant avoir eu une recherche officielle pour soutenir ce vécu!

Sans être exhaustif, attardons-nous à quelques constats observés au cours de ces années. Ces constats pourraient éventuellement être pris en considération dans l'amorce d'une réflexion collective, voire institutionnelle, menant à un changement de culture, ou du moins à son évolution, quant à une collaboration plus efficiente entre les différentes parties prenantes. 
Au fil de l'actualisation de ces différents rôles, il m'a été donné d'être aux confins des besoins, notamment d'ordre administratif, et des suivis inhérents à des demandes et projets de recherche émanant des milieux scolaires comme de ceux formulés, la plupart du temps, par les chercheurs universitaires eux-mêmes. Le praticien est un chercheur, au sens propre et figuré, qu'il soit enseignant, conseiller pédagogique ou gestionnaire. Il en sait parfois davantage sur l'objet de la recherche que le chercheur universitaire lui-même, qu'il soit en mesure ou non de l'expliciter et de le justifier en objectivant sa pratique. La recherche en éducation vient souvent légitimer en quelque sorte les bons coups existant dans les pratiques pédagogiques ou les pratiques de gestion. Le praticien et à fortiori ses élèves, seul ou en équipe, est au cœur de la situation de recherche. Malgré cela, il est rarement l'instigateur premier de la demande de recherche. Autrement dit, comment concilier ou prioriser les besoins exprimés par le milieu scolaire (domaines de l'enseignement, de l'accompagnement pédagogique et de la gestion du personnel) au regard des hypothèses de recherche du monde universitaire? Fondamentalement, la question est de savoir si la recherche répond aux besoins des praticiens ou des institutions (à savoir l'école et la commission scolaire), à ceux des chercheurs universitaires ou encore à ceux des deux parties. La combinaison des deux semble parfois déficiente, voire absente.

Quant à un espace de partage d'expériences et de collaboration entre les universités et les commissions scolaires, qui ont à la base comme dénominateur commun un besoin de recherche, la collaboration effective n'est pas nécessairement celle souhaitée en tout ou en partie par les deux instances, du moins en termes de pérennité, d'efficience, de retombées immédiates et de rayonnement. La plupart du temps, l'espace de partage et de collaboration entre les institutions ou entre les chercheurs et les praticiens est tributaire des modèles organisationnels et opératoires préconisés par les commissions scolaires. Ces modèles sont trop souvent dépendants des personnes concernées par les dossiers de la recherche universitaire, différenciés d'une organisation à l'autre et ne sont pas gages de stabilité dans le temps.

Un autre constat, davantage sous forme interrogative, ressort et semble prendre de l'importance. Pourquoi, au regard de toutes les recherches des trente dernières années, les pratiques pédagogiques, de gestion, organisationnelles, institutionnelles et communautaires (Daviau et St-Pierre, 2007) ont-elles si peu évolué au Québec ou du moins de façon inégale d'une région à l'autre ou d'une école à l'autre, pour ne pas dire d'une classe à l'autre? Peut-on modifier cette situation et y remédier? Beau sujet de recherche! On entend souvent de plus en plus l'expression « recherches probantes » pour inspirer et orienter les pratiques pédagogiques et les pratiques de gestion en milieu scolaire. Pourquoi des recherches qualifiées de probantes ont-elles peu de rayonnement? Pourquoi les résultats semblent-ils parfois extrêmement difficiles à implanter de manière à assurer un effet dans les milieux, par exemple pour tout ce qui concerne le maintien du redoublement ou encore les devoirs? Il y aurait lieu par exemple de s'inspirer des écoles qui ont mis fin aux devoirs, notamment les écoles alternatives, afin de permettre un réel échange sur ce sujet impliquant les parents et le personnel des écoles.

Par ailleurs, il est relativement aisé de constater que l'accès aux résultats de l'ensemble des recherches, toutes universités confondues, demeure problématique pour toutes les commissions scolaires. La communication, la consignation et l'accessibilité des résultats de recherche auprès de l'ensemble des chercheurs est une chose, mais son équivalent auprès du personnel des établissements et des commissions scolaires en est une autre. Savoir où chercher, et quoi chercher, pour le simple commun des mortels sur le plancher de l'école ou d'une commission scolaire, n'est pas nécessairement évident. Cela constitue 


\section{CHRONIOLE}

une certaine aberration au regard des moyens technologiques de communication actuels. Comment centraliser l'information autrement que par commission scolaire, quand cela se fait, et dépasser une certaine compétition entre chercheurs, parfois d'une même université, et universités? Toute proportion gardée, à une certaine époque pas si lointaine, une des meilleures sources d'informations et de réflexions, teintée de recherches, la plus utilisée par le personnel des commissions scolaires était la défunte revue Vie pédagogique. On y trouvait par moments des articles faisant état de recherches, mais aussi et surtout de l'information concernant ce qui se faisait en classe par les praticiens. Cette revue était un grand vecteur de ce qui se tramait en recherche, pour les praticiens, mais surtout une mine d'informations et de réflexions accessibles et vulgarisées. Il y a peut-être là de quoi s'inspirer comme modèle d'accès aux résultats des recherches des collègues universitaires, mais aussi à ceux des praticiens qui font l'école.

En conclusion, l'idée de dresser des ponts entre la recherche et le milieu scolaire, notamment par cette chronique, doit contribuer à permettre aux praticiens d'être accompagnés et soutenus afin de légitimer leurs bons coups en pédagogie et en gestion tout en permettant aux chercheurs de s'en inspirer. Il serait intéressant de poursuivre et d'approfondir la réflexion, tout en diversifiant et en multipliant les lieux et les moyens d'échanges concernant le modèle actuel de collaboration entre les universités et les commissions scolaires. Ainsi, les chercheurs pourraient faire ressortir et faire valoir les bons coups des praticiens, mais aussi et surtout rendre accessibles les résultats de recherches sur l'ensemble du territoire tout en fournissant des pistes d'implantation sur ce terrain fertile qu'est l'école.

\section{Références}

Daviau, C. et St-Pierre, M. (2007). Le renouvellement des pratiques de gestion et de formation des gestionnaires en contexte de réforme. Dans L. Lafortune, M. Ettayebi et P. Jonnaert (dir.), Observer les réformes en éducation (p. 97118). Sainte-Foy, QC : Presses de l'Université du Québec.

Fejzo, A., Dufour, F. et Daviau, C. (2018). Créer un espace de partage des expériences de collaboration entre les milieux universitaire et scolaire en vue de l'amélioration des pratiques pédagogiques et de gestion en éducation. Formation et profession, 26(2), 106-109. http://dx.doi.org/10.18162/fp.2018.a147

\section{Pour citer cet article}

Daviau, C. (2018) Avis de recherche. Formation et profession, 26(3), 109-111. http://dx.doi.org/10.18162/fp.2018.a156 